\title{
WEIGHTED NORMAL NUMBERS
}

Geon H. ChoE

We show that if $\left\{a_{k}\right\}_{k}$ is bounded then $\lim _{n \rightarrow \infty}(1 / n) \sum_{k=1}^{n} a_{k}(-1)^{d_{k}}=0$ for almost every $0 \leqslant x \leqslant 1$ where $x=\sum_{k=1}^{\infty} d_{k} 2^{-k}$ is the dyadic expansion of $x$. It is also shown that $(1 / n) \sum_{k=1}^{n} a_{k} \exp \left(2 \pi i \cdot p^{k} x\right) \rightarrow 0$ almost everywhere where $p>1$ is any fixed integer.

Let $(X, \mu)$ be a probability measure space. A measurable transformation $T: X \rightarrow$ $X$ is said to be measure preserving if $\mu\left(T^{-1} E\right)=\mu(E)$ for every measurable subset $E$. A measure preserving transformation $T$ on $X$ is called ergodic if $f(T x)=f(x)$, $f \in L^{1}(X, \mu)$, holds only for constant functions. Let $1_{E}$ be the indicator function of a measurable set $E$ and consider the behaviour of the sequence $\sum_{k=0}^{n-1} 1_{E}\left(T^{k} x\right)$ which equals the number of times that the points $T^{k} x$ visit $E$. The Birkhoff Ergodic Theorem implies that the relative frequency of the visits equals $\mu(E)$, that is,

$$
\lim _{N \rightarrow \infty} \frac{1}{N} \sum_{n=1}^{N} 1_{E}\left(T^{n} x\right)=\mu(E) .
$$

Consider the ergodic transformation $T: x \mapsto\{2 x\}$ on $[0,1)$, where $\{t\}$ is the fractional part of $t$. If $x=\sum_{k=1}^{\infty} d_{k} 2^{-k}$ is the dyadic expansion of $x$, then $d_{k}=1_{[(1 / 2), 1)}\left(T^{k-1} x\right)$. The same theorem applied to $T: x \mapsto\{2 x\}$ on $[0,1)$ gives the classical Borel's Theorem on normal numbers:

$$
\lim _{n} \frac{1}{n} \sum_{k=0}^{n-1} d_{k}=\frac{1}{2} \quad \text { almost everywhere, }
$$

hence almost every $\boldsymbol{x}$ is normal, that is, the relative frequency of the digit 1 in the binary expansion of $x$ is $1 / 2$. Equivalently we may rephrase it as $\lim _{n}(1 / n) \sum_{k=0}^{n-1}(-1)^{d_{k}}=0$

Received 20th October, 1994

Research partially supported by GARC-KOSEF.

Copyright Clearance Centre, Inc. Serial-fee code: 0004-9729/95 \$A2.00+0.00. 
almost everywhere, with respect to the Lebesgue measure. For general references, see $[6,7]$. For recent results on spectral properties of uniform distribution, see [2].

In this paper we obtain weighted ergodic theorems, in other words, we show that for some $T$ and a bounded sequence $\left\{a_{k}\right\}_{k}$ of complex numbers the limit of $(1 / n) \sum_{k=1}^{n} a_{k} f\left(T^{k} x\right)$ exists almost everywhere, if $f$ satisfies certain orthogonality conditions. Similar problems were studied by Nair [5] for the Gauss transformation $x \mapsto\{1 / x\}$ and sequences satisfying $a_{k} \in\{0,1\}$.

We need the following lemma. For the proof see Proposition 1.9 in [1].

LEMMA . Let $\left\{u_{j}\right\}_{j=1}^{\infty}$ be a bounded sequence of complex numbers and let $\left\{v_{j}\right\}_{j=1}^{\infty}$ be a sequence of complex numbers for which there exists a constant $M>0$ such that $(1 / n) \sum_{j=0}^{n-1}\left|v_{j}\right|^{2} \leqslant M$ for every $n$. Suppose that an increasing sequence of positive integers $\left\{N_{k}\right\}_{k=1}^{\infty}$ satisfies

(i) $\lim _{k \rightarrow \infty}\left(N_{k+1}\right) /\left(N_{k}\right)=1$,

(ii) $\left(1 / N_{k}\right) \sum_{j=0}^{N_{k}-1} u_{j} v_{j}$ converges to $A$ as $k \rightarrow \infty$.

Then $(1 / n) \sum_{j=0}^{n-1} u_{j} v_{j}$ also converges to $A$.

For a unitary operator $U$ in a Hilbert space $\mathcal{H}$ with the inner product $($,$) there$ exists a spectral measure $P$ such that $U=\int_{|z|=1} z d P(z)$ where $P(E)$ is an orthogonal projection in $\mathcal{H}$ for every measurable subset $E$. For $h \in \mathcal{H}$ we have a positive finite measure $\lambda_{h}$ such that $\lambda_{h}(E)=(P(E) h, h)$ and $\left(U^{n} h, h\right)=\int_{|z|=1} z^{n} d \lambda_{h}(z)$. But for a noninvertible measure preserving transformation $T$ acting on a probability space $(X, \mu)$ the induced linear operator $U_{T}$ in $L^{2}(X, \mu)$ defined by $\left(U_{T} f\right)(x)=f(T x)$ is not unitary, hence the spectral measure does not exist and the spectral theorem is not applicable.

Here is one of the ways to overcome this difficulty: Let $U$ be the isometry in $\mathcal{H}$ which is not necessarily invertible. Put $c_{n}=\left(U^{n} h, h\right)$ for $n \geqslant 0$ and $c_{n}=\left(\left(U^{*}\right)^{|n|} h, h\right)=\left(h, U^{|n|} h\right)$ for $n<0$, where $U^{*}$ is the adjoint of $U$. Then $c_{-n}=\overline{c_{n}}$ and the sequence $\left\{c_{n}\right\}_{n \in Z}$ is positive definite. Hence by Bochner's theorem there exists a positive finite measure $\lambda_{h}$ such that $c_{n}=\int_{|z|=1} z^{n} d \lambda_{h}(z)$ for $n \in \mathbb{Z}$. Note that $\left(U^{k} h, U^{j} h\right)=\left(U^{k-j} h, h\right)=c_{k-j}$ for $k \geqslant j$ and $\left(U^{k} h, U^{j} h\right)=\left(h, U^{j-k} h\right)=c_{k-j}$ for $k<j$. If there is an element $h \in \mathcal{H}$ such that $\left(U^{n} h, h\right)=0$ for every $n>0$, then $d \lambda_{h}$ and the normalised Lebesgue measure on the circle $d z$ have the same FourierStieltjes coefficients, hence we see that $d \lambda_{h}=C \cdot d z$ for $C=\|h\|^{2}$. For details on Bochner's theorem, see $[3,4]$. 
PROPOSITION 1. Let $\left\{a_{j}\right\}_{j}$ be a sequence of complex numbers such that

$$
\frac{1}{n} \sum_{j=0}^{n-1}\left|a_{j}\right|^{2} \leqslant M
$$

for every $n$. For almost every $0 \leqslant x \leqslant 1$ the limit of

$$
\frac{1}{n} \sum_{j=1}^{n} a_{j}(-1)^{d_{j}}
$$

exists and equals 0 where $x=\sum_{j=1}^{\infty} d_{j} 2^{-j}, d_{j} \in\{0,1\}$, is the dyadic expansion of $x$.

Proof: Let $T:[0,1) \rightarrow[0,1)$ be the Lebesgue measure preserving transformation given by $T x=\{2 x\}$. Let $h(x)=1_{[0,1 / 2)}(x)-1_{[1 / 2,1)}(x)=1-2 \cdot 1_{[1 / 2,1)}(x)$. Then $h\left(T^{j} x\right)$ is the $j$ th Rademacher function and $\left\{h\left(T^{j} x\right)\right\}_{j=0}^{\infty}$ is an orthonormal family in $L^{2}(0,1)$.

Hence the isometry $U_{T} f(x)=f(T x)$ satisfies for $j, k \geqslant 0$,

$$
c_{j-k}=\left(U_{T}{ }^{j} h, U_{T}{ }^{k} h\right)=\int_{0}^{1} h\left(T^{j} x\right) h\left(T^{k} x\right) d x=\delta_{j k}
$$

and

$$
\left(U_{T}^{j} h, h\right)=0 \text { for } j>0 \text {. }
$$

Since

$$
\begin{aligned}
\left\|\sum_{j=0}^{n-1} a_{j} U_{T}^{j} h\right\|^{2} & =\sum_{0 \leqslant j, k \leqslant n-1} a_{j} \overline{a_{k}} c_{j-k} \\
& =\sum_{j=0}^{n-1}\left|a_{j}\right|^{2} \leqslant n \cdot M,
\end{aligned}
$$

the Monotone Convergence Theorem implies that

$$
\begin{aligned}
\int_{0}^{1} \sum_{n=1}^{\infty}\left|\frac{1}{n^{2}} \sum_{j=0}^{n^{2}-1} a_{j} h\left(T^{j} x\right)\right|^{2} d x & =\sum_{n=1}^{\infty} \int_{0}^{1}\left|\frac{1}{n^{2}} \sum_{j=0}^{n^{2}-1} a_{j} h\left(T^{j} x\right)\right|^{2} d x \\
& =\sum_{n=1}^{\infty}\left\|\frac{1}{n^{2}} \sum_{j=0}^{n^{2}-1} a_{j} U_{T}{ }^{j} h\right\|^{2} \\
& \leqslant \sum_{n=1}^{\infty} \frac{1}{n^{4}} \cdot n^{2} \cdot M<\infty
\end{aligned}
$$


hence

$$
\sum_{n=1}^{\infty}\left|\frac{1}{n^{2}} \sum_{j=0}^{n^{2}-1} a_{j} h\left(T^{j} x\right)\right|^{2}<\infty \quad \text { almost everywhere }
$$

and

$$
\frac{1}{n^{2}} \sum_{j=0}^{n^{2}-1} a_{j} h\left(T^{j} x\right) \rightarrow 0 \quad \text { almost everywhere. }
$$

Putting $u_{j}=h\left(T^{j} x\right), v_{j}=a_{j}$ and $N_{k}=k^{2}$ we apply the Lemma. Then

$$
\frac{1}{n} \sum_{j=0}^{n} a_{j} h\left(T^{j} x\right) \rightarrow 0 \quad \text { almost everywhere. }
$$

Let $x=\sum_{j} d_{j} 2^{-j}$ be the dyadic expansion of $x$, and note that $d_{j}=1_{[(1 / 2), 1)}\left(T^{j-1} x\right)$ and use $h\left(T^{j-1} x\right)=1-2 \cdot 1_{[1 / 2,1)}\left(T^{j-1} x\right)=1-2 \cdot d_{j}(x)=(-1)^{d_{j}(x)}$.

REMARK. Let $p>1$ be a fixed integer. Using the Lebesgue measure preserving transformations $T x=\{p x\}, 0 \leqslant x \leqslant 1$ and the corresponding function $h$ defined by $h(x)=\exp ((2 \pi i(j-1) / p) x),(j-1) / p \leqslant x<j / p, j=1, \ldots, p$, we can easily see that for a bounded sequence $\left\{a_{k}\right\}_{k}$ of complex numbers the limit of

$$
\frac{1}{n} \sum_{k=1}^{n} a_{k} \lambda^{d_{k}} \quad \text { where } \lambda=\exp (2 \pi i / p)
$$

is equal to 0 almost everywhere, where $x=\sum_{k=1}^{\infty} d_{k} p^{-k}, d_{k} \in\{0,1, \ldots, p-1\}$ is the $p$-adic expansion of $x$.

Proposition 2. Let $\left\{a_{k}\right\}_{k}$ be a bounded sequence of complex numbers. For almost every $0 \leqslant x \leqslant 1$ we have

and

$$
\begin{aligned}
& \frac{1}{n} \sum_{k=1}^{n} a_{k} \sin \left(2 \pi i \cdot p^{k} x\right) \rightarrow 0 \\
& \frac{1}{n} \sum_{k=1}^{n} a_{k} \cos \left(2 \pi i \cdot p^{k} x\right) \rightarrow 0 \\
& \frac{1}{n} \sum_{k=1}^{n} a_{k} \exp \left(2 \pi i \cdot p^{k} x\right) \rightarrow 0
\end{aligned}
$$

where $p>1$ is a fixed integer.

Proof: Define $T x=\{p x\}, 0 \leqslant x \leqslant 1$. Note that the function $\exp (2 \pi i x)$ satisfies the condition $\left(U_{T}^{j} h, h\right)=0$ for $j>0$. Proceed as in Proposition 1 and take real and imaginary parts. 


\section{References}

[1] A. Bellow and V. Losert, "The weighted pointwise ergodic theorem and the individual ergodic theorem along subsequences', Trans. Amer. Math. Soc. 288 (1985), 307-345.

[2] G.H. Choe, 'Spectral types of uniform distribution', Proc. Amer. Math. Soc. 120 (1994), 715-722.

[3] H. Helson, Harmonic analysis (Addison-Wesley, 1983).

[4] Y. Katznelson, An introduction to Harmonic analysis (Dover, New York, 1976).

[5] R. Nair, 'On the metrical theory of continued fractions', Proc. Amer. Math. Soc. 120 (1994), 1041-1046.

[6] K. Petersen, Ergodic theory (Cambridge University Press, Cambridge London, 1983).

[7] P. Walters, An introduction to Ergodic theory (Springer-Verlag, New York, 1982).

Department of Mathematics

Korea Advanced Institute of Science and Technology

Taejon 305-701

Korea

e-mail: choe@euclid.kaist.ac.kr 